\title{
4 A Framework for Interactivity and Presence in Novel Bodies
}

\author{
Andrea Stevenson Won, Antal Haans, Wijnand A. IJsselsteijn, and \\ Jeremy N. Bailenson
}

\begin{abstract}
Researchers are beginning to explore the consequences of interacting with virtual worlds using non-human bodies. As virtual environments become more advanced, it is possible for participants to interact with their environments in increasingly sophisticated ways. Using trackers, users can control multiple avatar limbs in order to manipulate objects, move through space, and otherwise act in the virtual world. These avatar bodies need not conform to the normal human configuration, either in their appearance or in the way the tracked movements of the user are rendered to control the movements of the avatar. In this chapter we use the framework developed by Haans and IJsselsteijn to investigate the experience of presence in cases of nonhuman avatars or avatars that otherwise differ in ability or control schema from the user's own body; for example, avatars with more than two arms. We focus on cases where participants inhabit avatars in which the veridical mapping between tracking and rendering is disrupted.
\end{abstract}

Keywords: Homuncular Flexibility; Body Schema; Body Morphology, Body Image.

\subsection{Introduction to Chapter 4}

Media changes our behavior in the real world, and the way we understand media is based on our evolved responses to real world stimuli (Nass and Reeves, 1996). But what happens when our mediated experiences have no real world analogue- when we are experiencing life as a cow being raised for beef, or manipulating our environment with eight claws instead of two arms? How will we understand how to navigate in a body that is so dissimilar from the human, and how will our behavior be altered after such an experience?

One vision of virtual reality, in particular, looks to experiencing the impossible rather than duplicating the physical world. As Sutherland (1965) states in his classic "The Ultimate Reality", "There is no reason why the objects displayed by a computer have to follow the ordinary rules of physical reality with which we are familiar.” (p. 507). Classic virtual reality simulators were designed to allow users to experience environments, such as flight simulators, which were not physically available for reasons of expense or danger. As virtual systems advanced, it became possible for participants to interact with - and be represented within - these virtual environments in increasingly sophisticated ways. While it has become possible to customize avatars 
so that they resemble their controller, it is also possible to create avatars that are very different from their users' own bodies.

Social science research in virtual reality has leveraged this ability to alter the appearance avatars in order to give participants virtual self-representations which diverge from their real life appearance; changing gender, race, height, and level of attractiveness, among other qualities. Changing the appearance of a participant's avatar can lead to changes in the participant's behavior. This so-called "Proteus effect" has been demonstrated in a number of different experiments (Yee and Bailenson, 2006). In one experiment, participants who inhabited an avatar pre-rated as more than average attractiveness acted more socially and selected more attractive potential dating partners in a post-experiment task than participants who inhabited less attractive avatars. In another experiment, participants who inhabited a taller avatar acted more assertively post task. While this Proteus effect indicates that people adapt to and are affected by their avatars in a relatively short period of time, those avatars all conformed to the basic human template, and were controlled, to the extent that their gestures were incorporated, by tracking the natural movements of the participant and rendering them as accurately as possible in virtual reality.

However, it is also possible for people to inhabit and control nonhuman avatars, which may not follow the template of a primate walking on two legs and manipulating objects with his or her hands (Lanier, 2006; Kilteni, Normand, Sanchez-Vives \& Slater, 2012; Steptoe, Steed, \& Slater, 2013). This alters not only the relationship between how a participant appears in real life and how they appear in virtual reality, but also the relationship between a participant's tracked and rendered gestures. Potentially, then, people can experience presence in environments and in bodies that have never before been inhabited by humans in the physical world. However, they will understand these experiences based on their experiences with their human bodies, and the effects of these mediated experiences may resonate in the physical world as well.

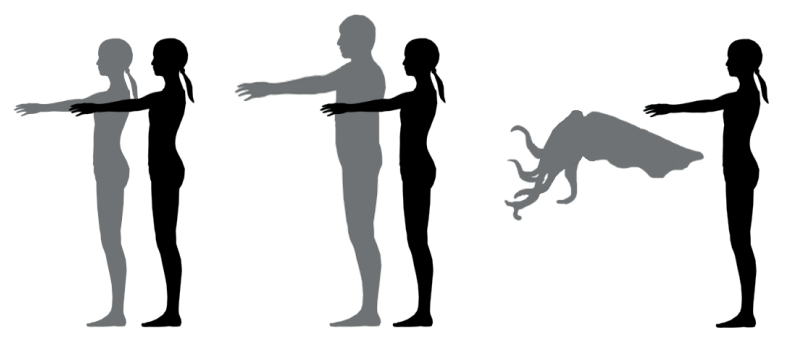

Figure 4.1: The leftmost figure shows a one-to-one relationship between the user (black silhouette) and her avatar (gray silhouette). The middle figure shows the Proteus effect, where the user controls an avatar that differs from her physical appearance, but the tracking/rendering relationship remains one-to-one, such that when the user raises her arm, her male avatar's arm is also raised. The rightmost figure shows a user controlling a non-human (cephalopod) avatar, where the rendered movement of the novel avatar cannot directly relate to the tracked movements of the user 
The investigation of presence has often been considered in the context of technological advances in media, although as Steuer (1992) points out in his discussion of virtual reality, it cannot be solely defined by hardware. Immersive virtual reality is a medium which makes the perceptual mediation of our sensory experiences very evident (Loomis, 1992). Thus, virtual reality has been a natural arena of research on the nature of presenceI, and is a logical medium in which to investigate presence in novel avatar bodies.

Presence is slippery to define and quantify. While some authors have linked presence to the affordances available from a given system, such as immersiveness and interactivity, others have defined it as the absence of awareness of technology, or the "perceptual illusion of non-mediation" (Lombard \& Ditton, 1997). One useful way to consider presence in the context of inhabiting an avatar is to follow Lee (2004) and break presence into three categories; environmental or spatial presence, social presence, and self-presence. We will focus primarily on self-presence when considering novel bodies. Self-presence may include the experience of not just being located in the virtual, but having a sense of ownership over it as well; feeling as if the virtual body is indeed your own (cf., Cole, Sacks, \& Waterman, 2000).

Self-presence has been further explicated (Riva, Waterworth, Waterworth, \& Mantovani, 2011; Ratan, 2012; Chapter 1 in this book) by being divided into three categories based on Damasio's theory of the self (1999). These categories, which are proto self-presence, core self-presence, and extended self-presence, relate to different areas of research on self-representation with avatars. Extended self-presence may be considered more related to an individual's identity, including the social construct of his or her personality, while core and proto self-presence appear to be more closely linked to the body as it affects and is affected by the surrounding environment. The way the body is understood in this context has to do with the sensation it receives from the environment, and the ability it has to act purposefully upon that environment. This leads to an understanding of presence as dependent on embodiment, where the latter is defined in Heideggerian terms as being an active participant in the world (also Zahorik \& Jenison, 1998). As Riva defines it, "presence is the intuitive perception of successfully transforming intentions into action (enaction).”

Understanding embodiment thus appears crucial for understanding presence, and in particular "presence" in atypical virtual bodies (see also Biocca, 1997; IJsselsteijn, 2004; Slater \& Usoh, 1994). Biocca, for example, argued that the body itself is not much different from the typical components of a virtual reality system: Both mediate the communication between the mind and the physical world. Indeed, as Loomis (1993) argued, any satisfactory theory of presence should be grounded in the understanding of ordinary sensorimotor processes. Thus, to explain how it is possible that people may learn to control a virtual body so different from their own, and may even come to consciously experience it as their own body, we must first have an understanding of how we are embodied in our natural bodies. One such theoretical framework was recently proposed by Haans and IJsselsteijn (2012). 
Based on the works of natural philosophers such as Metzinger $(2003,2006)$ and Gallagher (1986, 2005), Haans and IJsselsteijn (2012) describe the three different levels of human embodiment: morphology, a body schema, and a body image. In this theoretical framework, the body schema and image instigate a perceptual illusion of non-mediation; not with respect to media technology but to our own biological bodies. The body schema renders the workings of the body transparent to its owner; for example, allowing an individual to walk without having to pay attention to each individual muscle. Similarly, the body image is effectively a transparent self-model (Metzinger, 2009) which allows humans to make sense of the world as distinct from themselves, connecting past, presence and future, and allowing for the learning of refined bodily actions and offline simulations thereof. Both the body schema and image are highly flexible in accommodating technological tools as respectively functional and phenomenological extension of the body; allowing the same transparency in the use of tools as in using our natural bodies.

In the next sections we will use Haans and IJsselsteijn's framework for embodiment to interrogate the concept of presence in novel bodies. First we will examine the design, or morphology, of novel bodies. Next we will discuss tool use, and how this may relate to both embodiment broadly and the process of controlling avatar bodies. We will then examine the phenomenon of body transfer/body ownership, and how this relates to what changes in body image may be achievable when embodying novel avatars. Finally, we will look at the consequences of embodying novel avatars within the framework of embodied cognition.

\subsubsection{Extending Human Morphology In Novel Ways}

Morphology refers to the number, kind, and location of limbs, muscles, and sensory receptors. Our human morphology largely determines and constrains our action possibilities and perceptual experiences. For example, we lack the capacities for active flight or navigation by means of echolocation which are more typical of the bat morphology. Tools, including media technologies, may be used to extend the possibilities of our natural morphology, or to repair it in case of an accident. But what if, instead of simply extending our morphology, a person could become something else- a bat perhaps or an animal so far removed from the human that it does not even have the same kind of skeleton - an invertebrate, like a lobster?

Altering the physical appearance of the avatar, and changing how physical movements control it, using ipsimodal remapping, is only one aspect of potential novel avatar morphology changes. Sensory substitution, a remapping between two different sensory modalities, also provides the opportunity for novel avatar experiences. For example, visual information may be rendered as tactile stimuli; a technique used in the physical world in order to provide visually impaired people with information about their environment beyond the limits of touch (Bach-y-Rita \& W Kercel, 2003). 
Para-synthetic expression is another way of altering the morphology of an avatar body, by tracking input from the user which he or she may not be consciously aware of (such as psychological arousal, heart rate, or brain activity) and then rendering it so that it is easily observed (Janssen, Bailenson, IJsselsteijn \& Westerink, 2010). Further examples of novel remappings are described by Waterworth and Waterworth (Chapter 2, this volume).

However, mapping a new avatar body or sensory receptor onto our existing morphology is only the first step in establishing a sense of presence in that body. In order to control and interpret such a new mapping in a fluent and time efficient manner, the body schema should adapt to the new virtual body and / or the media technology that is used to map it into our existing one.

\subsection{Body Schema and Extending the Body Functionality with Tools}

Following Gallagher (1986), who defined the body schema "as the unconscious performance of the body” (p. 548), Haans and IJsselsteijn (2012) propose a definition of the body schema as a dynamic distributed network of procedures aimed at guiding behavior. One important aspect of the body schema is that these procedures can largely operate outside of our conscious awareness; for example, being involved in distance estimation, action selection, muscle activation, keeping balance and other abilities that allow us to control our bodies in a fluent manner. In other words, having a body schema allows us to use the various parts of our complex morphology as a coherent functional unity. A second important aspect of the body schema is that it is highly adaptive, not only in accommodating the natural growth of the body, but in incorporating technological artifacts as functional extensions of the body.

\subsubsection{Tool Use}

One avenue of research on tool use has examined whether and how tools may be incorporated into the body schema--or alternatively phrased (see also Chapter 1 , this volume): whether and how the network of body schema procedures adapts to the use of a tool. One example is Berti and Frassinetti's (2000) research on a patient (P.P) suffering from left-sided near space neglect. When a line was presented in far space, she performed equally well on a line bisection task as healthy controls. However, when pointing to the perceived midpoint of the line using a stick rather than a laser pointer, her near space neglect suddenly shifted into far space. This suggests that the brain remaps the space around the body to accommodate for the expansion of our reaching space when using a stick as a tool. Other research suggests that representations of the arm, as part of the dynamic body schema, are similarly adaptive and may become elongated when using tools that increase our reaching space. Cardinali and colleagues 
(2009),for example, asked blindfolded participants to indicate various positions on their right arm with their other hand before and after using a grabber. Their results show an increase in distance between the indicated position of fingertips and the elbow as a result of tool use.

While the nature of the changes imposed by tool use remains subject to debate (e.g., Holmes, 2012), the ability of users to alter their behavior in order to interact with the environment through the medium of some tool is relevant to adaptation to novel avatar bodies. As described in Riva \& Mantovani (2012 ; Chapter 1, this volume), the way people learn to manipulate an environment using tools shapes both their understanding of space and their sense of presence therein. As Haans and IJsselsteijn (2012) point out, adapting to the sensory feedback provided by tools is directly analogous to adapting to the feedback provided by immersive media technologies, and thus by inference, when interacting with a simulated environment by means of a virtual body. Thus when controlling an avatar, as with tool use, the user has the opportunity to actually enact movements that are not possible in real life with the unassisted human body.

Observers are not able to intuitively understand anatomically impossible movements by avatars when observing them from the third person perspective (Borroni, Gorini, Riva, Bouchard \& Cerri, 2011). However, allowing users to create these impossible movements from a first person perspective in a way that imitates the mediation offered by normal sensory input raises very interesting questions of how they may incorporate such movements into the body schema, as some aspects of body schema development appear to be affected by experience and agency.

It is this potential flexibility of the body schema that led Jaron Lanier to choose the term "homuncular flexibility" to describe users' adaptability to novel bodies in virtual reality. The name reflects Lanier's proposal that the "homunculi" of the somatosensory and motor cortices- the portions of the brain described by Penfield and Boldrey (1937) that roughly map areas of bodily sensation and motor control- can be altered based on sensory input such as that provided by virtual reality. "Flexibility" refers to the idea that controlling an avatar body from the first person perspective without using a tool- that is, by using body movements alone and not manipulating a secondary object- is a very special example of tool use.

When considering presence in novel bodies, tool use probably provides the best analogy to consider how a feeling of presence may be created. With homuncular flexibility, presence is the expected result of learning to control an avatar body through the degrees of freedom available in the user's physical body without requiring that the avatar match the user, either in appearance or in the method of control. However, to feel present in this atypical avatar, the workings of that new body, and thus the way in which it is mapped upon the original one, should become transparent to its user. Within the framework of Haans and IJsselsteijn (2012) this requires that the body schema can adapt to include the components of the new body. 
Presence in an avatar, however, means more than being present in the location of the avatar. It may also involve the experience that the avatar body is indeed your own; self-presence rather than spatial presence. This involves not just incorporation in the body schema, but in the body image as well.

\subsection{Body Image and the Transfer of Body Ownership to Novel Bodies}

In the framework by Haans and IJsselsteijn (2012), the body image is a product of consciousness. According to Edelman (2003; 2006) consciousness is the result of neural processes that allow for a large amount of refined discriminations and perceptual categorizations ranging from, for example, the "redness" of red to the unitary perceptual scene, emotions, and memories alike. The body image, as defined by Haans and IJsselsteijn, consists of those discriminations that pertain to the individual's own body (i.e., to those objects that the central nervous system has categorized as being a part of the physical body). In contrast to the body schema, the body image requires a notion of the body as owned by the user (see Gallagher, 1986).

This sense of body ownership may at times be transferred to tools as well, including the arms of a virtual avatar or teleoperation robot. Such incorporations into the body image are conceptually different from body schema incorporations: Body schema incorporations allow for proficient use of tools (i.e., as functional extensions of our morphology), but do not require a transfer of body ownership toward that tool (i.e., a phenomenological extension in which the tool becomes part of our bodily selves). The extent to which virtual avatars that do not correspond to the natural human body can be incorporated into the body image remains to be discovered.

\subsubsection{Body Transfer and Body Ownership}

While body transfer has been studied in and out of virtual reality, it began as investigations in the real world. In the rubber hand illusion, first demonstrated by Botvinick and Cohen (1998), participants are seated in front of a table on which an obviously fake rubber hand is placed in approximately the orientation of their real hand. Their own real hand is placed out of sight, either under the table or behind a screen. Subsequently both hands are simultaneously and synchronously stroked with a soft brush. After a few minutes, many participants report a sense of body ownership over the fake hand, and when the rubber hand is suddenly struck with a rubber mallet, many react violently, as if their own hand had been struck (Armel \& Ramachandran, 2003). This work has been extended in virtual reality. IJsselsteijn, de Kort and Haans (2006) replicated the rubber hand illusion in physical, virtual and mixed reality, demonstrating similar effects although the strength of the subjective illusion differed (see also Slater, Perez-Marcos, Ehrsson, \& Sanchez, 2008). 
Research on the rubber-hand illusion has revealed that body ownership results from establishing so-called body specific sensorimotor contingencies (Botvinick, 2004). The clapping of one's hands, whether self-initiated or not, consists of correlated multisensory impressions. Some of these patterns of sensorimotor contingencies are exclusively associated with the body, and hence self-specifying: When the visual image of clapping hands is accompanied immediately by a tactile sensation in the hands, then by inference it must be your hands that do the clapping. In the rubber hand illusion, some of these body specific contingencies are mapped onto the fake hand. At the same time, the incorporation of the fake hand into the body image appears to be modulated top-down by an internal model of what the human body is like: Objects that differ morphologically from the human body are less easily incorporated (e.g., Haans, IJsselsteijn, \& de Kort, 2008; de Vignemont, Tsakiris, \& Haggard, 2006; Tsakiris \& Haggard, 2005).

The rubber hand illusion has been extended by, among others, Petkova and Ehrsson (2008), who investigated whole body transfer, including establishing a sense of ownership in a mannequin body, and switching the gender of the body from the participants' own gender. In addition, work on "body transfer” in virtual reality further supports the idea that people can be brought to identify with avatars that differ in appearance and gender (Slater, Spanlang, Sanchez-Vives \& Blanke, 2010). Such experiments show results similar to the Proteus effects shown by first-person perspective in a mirror condition. Consistent with the rubber hand illusion research, Petkova and Ehrsson demonstrate that the body had to be human-like in order for body ownership to transfer. However, some work has been done than indicates that disruption of the normal human template does not preclude this effect. For example, Schaefer, Flor, Heinze and Rotte (2007) created a sense of ownership in a very long arm by simultaneous touch, and Kilteni, Normand, Sanchez-Vives and Slater, (2012) duplicated this phenomenon in a virtual environment.

In the experiment by Kilteni et al (2012) participants were able to transfer ownership to an extremely long virtual arm extending up to three to four times the length of the participant's own; showing thus little of the top-down modulation consistently found in the rubber hand illusion literature (cf. Armel and Ramachandran, 2013). The authors argue that this top-down modulation may perhaps be countered by increasing the richness of the multisensory stimulation, which in their experiment involved not just establishing visuotactile contingencies, but visuomotor, and tactilemotor contingencies as well. Recent evidence on the rubber-hand illusion supports this claim by demonstrating that increasing the amount of information in the visuotactile stimulation (e.g., tapping versus stroking the fingers) makes it easier for people to experience the illusion (Haans, Kaiser, Bouwhuis, \& IJsselsteijn, 2012).

Body transfer or body ownership emphasizes the role of simultaneous, synchronous multisensory input over taking action in a virtual environment. While much of the experimental work has examined variations of the normal human 
template, the potential exists for body transfer to occur in bodies that stretch the definition of the normal.

Creating a sense of ownership over novel bodies not only requires the establishment of new body-specific sensorimotor contingencies, but the morphological discrepancies between the novel body and its human counterpart are more extensive as well. Developing a sense of ownership over a novel body may be more difficult than establishing it over a human-like body or body part. Rich multisensory stimulation may help to successfully transfer ownership to a novel body--realizing of course that action allows for richer sensorimotor interactions then exclusively passive stimulation. Also, such ownership transferal may not come instantaneously. While the original definition of homuncular flexibility specifically referred to the alteration of a map of the body over the very short term (the minutes in which a person could inhabit an avatar in virtual reality) considerable time and practice with using a novel morphology may be required before such a morphology may be incorporated in the body schema and image. At the same time, we should not forget the possibility that the plasticity of our human embodiment may have limits, for example because of congenital factors (for a discussion, see Price, 2006).

\subsection{Embodied Cognition and Virtual Environments}

Embodied cognition proposes that people understand the world through their bodies. In other words, not only does the mind guide and prompt the body to action, but the actions the body takes and the resulting experiences, shape how a person comes to understand not only his- or herself, but the surrounding environment (Wilson, 2002; Anderson, 2003). For example, most people are right-handed and can complete tasks more easily using their dominant hand. Embodied cognition researchers propose that right-handed people thus develop a preference for the right side of their world, such that objects that are placed on the right side of a person's body are viewed more positively than those on the left (thus the etymology of the word "sinister" which means, to the left (Casasanto \& Chrysikou, 2011).

Embodied cognition is a particularly useful concept when considering presence in a non-human avatar because it takes into account the effects that such an avatar may have on the user's ability to understand and manipulate the virtual environment. If people inhabit an environment in a body with different affordances, that experience may affect their understanding of both that environment, and possibly that of the physical world to which they return.

Some work on embodied cognition does imply that this influence can be changed rather rapidly, as in an experiment by Casasanto and Chrysikou (2011) where right-handed people were temporarily handicapped by wearing a ski glove on their right hand, such that their left hand was temporarily more able than their right. Participants then showed the same association as natural left-handed people 
in assigning "good" objects to the left and "bad" objects to the right. Clark (2007) similarly emphasizes the role of activity when he describes the body as a "suite of potentials for action" in which tools can augment and extend the body. This concept reinforces the idea that the extent to which a user may interact with the virtual world using a novel body may alter the extent to which said user feels truly present in that body and that world.

Research in embodied cognition would thus imply that relatively short interventions affecting an individual's ability to take action in the environment will result in changes in how that individual perceives that environment and how present they feel. Altering the appearance and functionality of an avatar may also lead to changes in how the virtual environment is perceived. When a person operates an avatar with multiple, elongated limbs, far may become near. If a person's legs become much more fluent than his or her arms, perhaps the positive valence for higher objects may reverse. However, many of the associations that an adult holds for his or her body have been in existence since birth and augmented by experience. The extent to which these long-standing associations may be altered by a brief experience in an avatar body remains to be seen.

\subsection{Conclusion to Chapter 4}

As virtual reality has developed, the worlds and experiences available therein have continued to diverge from the real. In addition, the ability of users to interact with virtual environments and effect changes in these virtual worlds has been augmented as technology improves. More naturalistic gestures expand the situations in which users may or may not experience a sense of presence. In addition, methods of tracking human gestures that are much less obtrusive will probably continue to proliferate. Current efforts to use interfaces that do not require the user to wear any markers or hold any devices (for example, the Microsoft Kinect) point the way to a future in which movements can be captured in an everyday environment and be used to drive mediated interactions.

Investigating avatar design can provide concrete evidence for ways of improving user interfaces. Aspects of design decisions that are often made intuitively can have effects on users' understanding of their bodies and their interactions with their environment. In addition, virtual reality offers the opportunity to change the world and the body, and examine the resulting effects on the self, in ways that are not possible in real life. This allows us to manipulate embodiment to change outcomes, attitudes, and learning.

Virtual reality allows us to see explicitly the affordances of our mediated bodies. However, our abilities are being changed by current technologies in both virtual reality and real life, as we see devices proliferate that provide increased memory, ability to access group knowledge instantly, etc. This illustrates Biocca's “cyborg's 
dilemma" (Biocca, 1992) as we consider how extending our sense of embodiment, by augmenting the mediated ways in which we may control our environment, may be changing our fundamental sense of our selves.

\section{References}

Anderson, M. L. (2003). Embodied Cognition: A Field Guide. Artificial Intelligence, 149, 91-130.

Armel, K. C. \& Ramachandran, V. S. (2003). Projecting sensations to external objects: Evidence from skin conductance response. Proceedings of the Royal Society of London B, 270, 1499-1506.

Bach-y-Rita, P., \& W Kercel, S. (2003). Sensory substitution and the human-machine interface. Trends in cognitive sciences, 7(12), 541-546.

Berti, A., \& Frassinetti, F. (2000). When far becomes near: re-mapping of space by tool use.

Biocca, F. (1999). The cyborg's dilemma: Progressive embodiment in virtual environments. Human Factors in Information Technology, 13, 113-144.

Borroni, P., Gorini, A., Riva, G., Bouchard, S., \& Cerri, G. (2011). Mirroring avatars: dissociation of action and intention in human motor resonance. European Journal of Neuroscience, 34(4), 662669.

Botvinick, M., \& Cohen, J. (1998). Rubber hands' "feel” touch that eyes see. Nature, 391(6669), 756756.

Botvinick, M. (2004). Probing the neural basis of body ownership. Science, 305, 782-783.

Carlson, T. A., Alvarez, G., Wu, D. A., \& Verstraten, F. A. (2010). Rapid assimilation of external objects into the body schema. Psychological Science, 21(7), 1000-1005.

Cardinali, L., Frassinetti, F., Brozzoli, C., Urquizar, C., Roy, A. C., \& Farnè, A. (2009). Tool-use induces morphological updating of the body schema. Current Biology, 19(12), 478-479

Casasanto, D., \& Chrysikou, E. G. (2011). When left is “right” motor fluency shapes abstract concepts. Psychological Science, 22(4), 419-422.

Clark, A. (2007). Re-inventing ourselves: The plasticity of embodiment, sensing, and mind. Journal of Medicine and Philosophy, 32(3), 263-282.

Cole, J., Sacks, O., \& Waterman, I. (2000). On the immunity principle: A view from a robot. Trends in Cognitive Science, 4, 167.

Damasio, A. (1999). The feeling of what happens: body, emotion and the making of consciousness. San Diego: Harcourt Brace.

de Vignemont, F., Tsakiris, M., \& Haggard, P. (2006). Body mereology. In G. Knoblich, I. M. Thornton, M. Grosjean, \& M. Shiffrar (Eds.), Human body perception from the inside out (pp. 147-170). New York: Oxford University Press.

Edelman, G. M. (2003). Naturalizing consciousness: A theoretical framework. Proceedings of the Natural Academy of Sciences of the United States of America, 100, 5520-5524.

Edelman, G. M. (2006). Second nature: Brain science and human knowledge. New Haven, CT: Yale University Press.

Ehrsson, H. H. (2007). The experimental induction of out-of-body experiences. Science, 317, 10481048.

Gallagher, S. (1986). Body image and body schema: A conceptual clarification. Journal of Mind and Behavior, 7, 541-554.

Gallagher, S. (2005). Dynamic models of body schematic processes. In H. de Preester, V. Knockaert (Eds.), Body image and body schema (pp. 233-250.). Amsterdam: John Benjamins.

Haans, A., \& IJsselsteijn, W. A. (2012). Embodiment and telepresence: Toward a comprehensive theoretical framework. Interacting with Computers, 24(4), 211-218. 
Haans, A., \& IJsselsteijn, W. A., \& de Kort, Y. A. W. (2008). The effect of similarities in skin texture and hand shape on perceived ownership of a fake limb. Body Image: An International Journal of Research, 5, 389-394.

Haans, A., Kaiser, F.G., Bouwhuis, D.G. \& IJsselsteijn, W.A. (2012). Individual differences in the rubber-hand illusion: Predicting self-reports of people's personal experiences. Acta Psychologica, 141, 169-177.

Holmes, N. P. (2012). Does tool use extend peripersonal space? A review and re-analysis. Experimental brain research, 218(2), 273-282.

IJsselsteijn, W. A. (2005). Towards a neuropsychological basis of presence, Annual Review of CyberTherapy and Telemedicine, 3, 25-30.

IJsselstein, W. A., de Kort, Y. A., \& Haans, A. 2006. Is this my hand I see before me? The rubber hand illusion in reality, virtual reality and mixed reality. Presence, 15: 455-464.

Janssen, J.H., Bailenson J.N., IJsselsteijn, W.A., \& Westerink, J.H.D.M. (2010). Intimate heartbeats: Opportunities for affective communication technology. IEEE Transactions on Affective Computing, 1(2), 72-80.

Kilteni, K., Normand, J-M., Sanchez-Vives, M. V., \& Slater, M. (2012). Extending Body Space in Immersive Virtual Reality: A Very Long Arm Illusion. PLoS ONE 7(7): e40867.

Lanier, J. (2006). Homuncular Flexibility, http://www.edge.org/response-detail/11182print.html lanier. Edge Foundation, Inc. Accessed February 3, 2014.

Lee, K. M. (2004). Presence, explicated. Communication theory, 14(1), 27-50.

Lombard, M., \& Ditton, T. (1997). At the heart of it all: The concept of presence. Journal of Computer Mediated Communication, 3(2), 0-0.

Longo, M. R., \& Lourenco, S. F. (2006). On the nature of near space: Effects of tool use and the transition to far space. Neuropsychologia, 44(6), 977-981.

Loomis, J. M. (1992). Distal attribution and presence. Presence: Teleoperators and Virtual Environments, 1(1), 113-119.

Loomis, J. M. (1993). Understanding synthetic experience must begin with the analysis of ordinary perceptual experience. In Proceedings of the IEEE Symposium on Research Frontiers in Virtual Reality (pp. 54-57). Los Alamos, CA: IEEE Press.

Maselli, A., \& Slater, M. (2013). The building blocks of the full body ownership illusion. Frontiers in human neuroscience, 7 (83), 1-20.

Metzinger, T. (2003). Being no one: The self-model theory of subjectivity. Cambridge, MA: MIT press.

Metzinger, T. (2006). Reply to Gallagher: Different conceptions of embodiment. Psyche 12(4), 1-7.

Metzinger, T. (2009). The ego tunnel: The Science of the mind and the myth of the self. New York: Basic Books.

Nass, C., \& Reeves, B. (1996). The Media Equation: How People Treat Computers, Televisions, and New Media as Real People and Places. Stanford, Calif.: CSLI Publications ; New York: Cambridge University Press.

Penfield, W., Boldrey, E. (1937) Somatic motor and sensory representation in the cerebral cortex of man as studied by electrical stimulation. Brain, 60, 389-443.

Petkova, V. I., \& Ehrsson, H. H. (2008). If I were you: perceptual illusion of body swapping. PloS one, 3(12): e3832.

Price, E. H. (2006). A critical review of congenital phantom limb cases and a developmental theory for the basis of body image. Consciousness and Cognition, 15(2), 310-322.

Ratan, R. A. (2012). Self-presence, explicated: Body, emotion, and identity extension into the virtual self. In R. Luppicini (Ed.), Handbook of Research on Technoself. New York, NY: IGI Global.

Riva, G., \& Mantovani, F. (2012). From the body to the tools and back: a general framework for presence in mediated interactions. Interacting with Computers, 24(4), 203-210. doi: doi: 10.1016/j.intcom.2012.04.007 
Riva, G., Waterworth, J. A., Waterworth, E. L., \& Mantovani, F. (2011). From intention to action: The role of presence. New Ideas in Psychology, 29(1), 24-37.

Schaefer, M., Flor, H., Heinze, H. J., \& Rotte, M. (2007). Morphing the body: illusory feeling of an elongated arm affects somatosensory homunculus. Neuroimage, 36(3), 700-705.

Slater M., Perez-Marcos D., Ehrsson H.H., Sanchez-Vives M.V. (2008) Towards a digital body: The virtual arm illusion. Frontiers in Human Neuroscience, 2(6), 1-8.

Slater, M., Spanlang, B., Sanchez-Vives, M. V., \& Blanke, O. (2010). First person experience of body transfer in virtual reality. PloS one, 5(5): e10564.Slater, M. \& Usoh, M. (1994). Body centred interaction in immersive virtual environments, in: N. Magnenat Thalmann, D. Thalmann (Eds.), Artificial life and virtual reality (pp. 125-148). Chichester, UK: John Wiley.

Steptoe, W., Steed, A., \& Slater, M. (2013). Human Tails: Ownership and Control of Extended Humanoid Avatars. Visualization and Computer Graphics, IEEE

Transactions on, 19(4), 583-590.Steuer, J. (1992). Defining virtual reality: Dimensions determining telepresence. Journal of communication, 42(4), 73-93.

Sutherland, I. E. (1965). The ultimate display. Multimedia: From Wagner to virtual reality. Proceedings of the IFIP Congress, 506-508.

Tsakiris, M., \& Haggard, P. (2005). The rubber hand illusion revisited: Visuotactile integration and self-attribution. Journal of Experimental Psychology. Human Perception and Performance, 31, 80-91.

Wilson, M. (2002). Six views of embodied cognition. Psychonomic bulletin \& review, 9(4), 625-636.

Yee, N., \& Bailenson, J. (2007). The Proteus effect: The effect of transformed self-representation on behavior. Human communication research, 33(3), 271-290.

Zahorik, P., \& Jenison, R.L. Presence as being-in-the-world. Presence-Teleoperations and Virtual Environments, 7, 78-89. 